\title{
Development of MITC isotropic triangular shell finite elements
}

\author{
Phill-Seung Lee, Klaus-Jürgen Bathe * \\ Massachusetts Institute of Technology, Cambridge, MA 02139, USA \\ Received 11 October 2003; accepted 28 January 2004
}

\begin{abstract}
We present a simple methodology to design isotropic triangular shell finite elements based on the Mixed Interpolation of Tensorial Components (MITC) approach. Several mixed-interpolated isotropic triangular shell finite elements are proposed. We perform well-established numerical tests and show the performance of the new elements.

(c) 2004 Published by Elsevier Ltd.
\end{abstract}

Keywords: Shell structures; Finite elements; Triangular elements; MITC shell elements

\section{Introduction}

It is well known that a shell structure is one of the most effective structures which exist in nature. Also, there exist countless man-made shell structures, which have been constructed in the human's history. To analyze shell structures, shell finite elements have been developed for several decades and have been used abundantly [1,2].

Shell structures can show different sensitivities with decreasing thickness, depending on the shell geometry and boundary conditions. As the thickness becomes small, the behavior of a shell structure belongs to one of three different asymptotic categories: the membranedominated, bending-dominated, or mixed shell problems [2-5]. An ideal finite element formulation should uniformly converge to the exact solution of the mathematical model irrespective of the shell geometry, asymptotic category and thickness. In addition, the convergence rate should be optimal.

As is well known, displacement-based shell finite elements are too stiff for bending-dominated shell structures when the shell is thin, regardless of the displacement interpolation order. In other words, the convergence of the element formulation in bending-

\footnotetext{
${ }^{*}$ Corresponding author. Tel.: +1-617-253-6645; fax: +1-617253-2275.

E-mail address: kjb@mit.edu (K.J. Bathe).
}

dominated problems deteriorates significantly as the ratio of the shell thickness to characteristic length $(t / L)$ decreases. This dependency of the element behavior on the thickness parameter is called "shear and membrane locking", which is the main obstacle in the finite element analysis of shell structures.

The "mixed interpolation of tensorial components" (MITC) approach has been used as a very successful locking removal technique for quadrilateral plate/shell finite elements. The technique was originally proposed for 4-node and 8-node shell elements (the MITC4 and MITC8 elements) by Dvorkin and Bathe [6,7] and was later extended to 9 and 16-node elements (the MITC9 and MITC16 elements) by Bucalem and Bathe, see Ref. [8]. The technique was also used for triangular plate and shell elements [9-12] and in particular regarding shell analyses shows further potential.

The main topic in shell finite element analyses is focused on answering the question "Is a given shell finite element uniformly optimal for general shell structures?". The recent studies [11-14] showed how to evaluate the optimality of shell finite elements and the studies reported that the mixed shell finite elements using the MITC technique are close to optimal in discretizations using quadrilateral shell finite elements.

When modeling general engineering structures, some triangular elements are invariably used. Indeed, triangular elements are most efficient to discretize arbitrary shell geometries. However, for quadrilateral shell finite element discretizations more research effort has been 
undertaken and more progress has also been achieved. Consequently, in shell finite element analyses, quadrilateral elements are usually used due to their better performance than observed using triangular elements. Indeed, there does not exist yet a "uniformly optimal" triangular shell element, and not even an element close to optimal. The motivation of this research comes from the fact that the development of optimal triangular shell elements is still a great challenge [10,12,15-19].

It is extremely difficult to obtain a shell finite element method that is uniformly optimal and a mixed formulation must be used. In the formulation we should aim to satisfy $[1,2]$ :

- Ellipticity. This condition ensures that the finite element discretization is solvable and physically means that there is no spurious zero energy mode. Without supports, a single shell finite element should havefor any geometry - exactly six zero energy modes corresponding to the physical rigid body modes. This condition can be easily verified by counting the number of zero eigenvalues (and studying the corresponding eigenvectors) of the stiffness matrix of single unsupported shell finite elements.

- Consistency. Since the finite element discretization is based on a mathematical model, the finite element solutions must converge to the solution of the mathematical model as the element size $h$ goes to zero. In other words, the bilinear forms used in the finite element discretization, which may be a function of the element size $h$, must approach the exact bilinear forms of the mathematical model as $h$ approaches zero.

- Inf-sup condition. Ideally, a mixed finite element discretization should satisfy the inf-sup condition $[2,11,13]$. For shell finite elements, satisfying this condition implies uniform optimal convergence in bending-dominated shell problems. Then, the shell finite element is free from shear and membrane locking with solution accuracy being independent of the shell thickness parameter. However, it is generally not possible to analytically prove whether a shell finite element satisfies this condition and numerical tests have been employed.

For triangular shell elements, one more requirement exists; namely, "spatial isotropy". The requirement of "spatial isotropy" means that the element stiffness matrices of triangular elements should not depend on the sequence of node numbering, i.e. the element orientation. Specifically, when a spatially isotropic triangular element has sides of equal length, the internal element quantities should vary in the same manner for each corner nodal displacement/rotation and each midside nodal displacement/rotation, respectively. If the behavior of an element depends on its orientation, spe- cial attention must be given to the direction of each element in the model.

In fact, this condition is a major obstacle in the construction of locking-free triangular shell elements. Usually, some "averaging" or "cyclic" treatments are employed to construct isotropic triangular elements $[18,19]$. A simple systematic way, which is mechanically clear, to construct isotropic triangular shell elements is desirable.

If a triangular shell element satisfies all the above conditions, it is an optimal and ideal triangular shell finite element. Such an element is very difficult to reach and we can "soften" the requirements somewhat for practical purposes. We summarize the practical requirements on triangular shell finite elements as follows:

- spatially isotropic behavior;

- no spurious zero energy mode (ellipticity condition);

- no shear locking in plate bending problems;

- reliable, ideally optimal results for membrane dominated shell problems;

- reliable, ideally optimal results for bending dominated shell problems "in the practical range of $t / L$ ";

- easy extension of the formulation to nonlinear analyses (simple formulation).

Hence only a practical range $t / L$ is considered in bending dominated shell problems (but, of course, we shall not abolish the aim to ultimately reach a triangular element that is optimal for all analyses and for all values of $t / L$ ).

The objective of this paper is to develop MITC isotropic triangular shell finite elements which can be practically used for general shell structures.

In the following sections of the paper, we first review the MITC formulation of continuum mechanics based shell finite elements. We then propose a simple methodology to design isotropic triangular shell elements using the MITC technique, and demonstrate this methodology with some examples. A large number of elements can be constructed using our methodology. We introduce some selected MITC triangular shell finite elements and give the numerical test results of these elements.

\section{MITC formulation of continuum mechanics based shell finite elements}

The continuum mechanics displacement-based shell finite elements have been proposed as general curved shell finite elements [20]. While these elements offer significant advantages in the modeling of arbitrary complex shell geometries, they exhibit severe locking in bending dominated cases $[1,2]$. 
The basic idea of the MITC technique is to interpolate displacements and strains separately and "connect" these interpolations at "tying points". The displacement and strain interpolations are chosen so as to satisfy the ellipticity and consistency conditions, and as closely as possible the inf-sup condition.

The geometry of the $q$-node continuum mechanics displacement-based shell element is described by $[1,2]$

$\vec{x}(r, s, t)=\sum_{i=1}^{q} h_{i}(r, s) \vec{x}_{i}+\frac{t}{2} \sum_{i=1}^{q} a_{i} h_{i}(r, s) \vec{V}_{n}^{i}$,

where $h_{i}$ is the $2 \mathrm{D}$ shape function of the standard isoparametric procedure corresponding to node $i, \vec{x}_{i}$ is the position vector at node $i$ in the global Cartesian coordinate system, and $a_{i}$ and $\vec{V}_{n}^{i}$ denote the shell thickness and the director vector at node $i$, respectively. Note that in this geometric description the vector $\vec{V}_{n}^{i}$ is not necessarily normal to the shell midsurface.

The displacement of the element is given by

$\vec{u}(r, s, t)=\sum_{i=1}^{q} h_{i}(r, s) \vec{u}_{i}+\frac{t}{2} \sum_{i=1}^{q} a_{i} h_{i}(r, s)\left(-\vec{V}_{2}^{i} \alpha_{i}+\vec{V}_{1}^{i} \beta_{i}\right)$

in which $\vec{u}_{i}$ is the nodal displacement vector in the global Cartesian coordinate system, $\vec{V}_{1}^{i}$ and $\vec{V}_{2}^{i}$ are unit vectors orthogonal to $\vec{V}_{n}^{i}$ and to each other, and $\alpha_{i}$ and $\beta_{i}$ are the rotations of the director vector $\vec{V}_{n}^{i}$ about $\vec{V}_{1}^{i}$ and $\vec{V}_{2}^{i}$ at node $i$.

The covariant strain components are directly calculated by

$e_{i j}=\frac{1}{2}\left(\vec{g}_{i} \cdot \vec{u}_{j}+\vec{g}_{j} \cdot \vec{u}_{i}\right)$,

where

$\vec{g}_{i}=\frac{\partial \vec{x}}{\partial r_{i}}, \quad \vec{u}_{i}=\frac{\partial \vec{u}}{\partial r_{i}} \quad$ with $\quad r_{1}=r, r_{2}=s, r_{3}=t$.

Now we define a set of so-called tying points $k=$ $1, \ldots, n_{i j}$ on the shell midsurface with coordinates $\left(r_{i j}^{k}\right.$, $s_{i j}^{k}$ ), and define the assumed covariant strain components $\tilde{e}_{i j}$ as

$\tilde{e}_{i j}(r, s, t)=\left.\sum_{k=1}^{n_{i j}} h_{i j}^{k}(r, s) e_{i j}\right|_{\left(r_{i j}^{k}, s_{i j}^{k}, t\right)}$,

where $n_{i j}$ is the number of tying points for the covariant strain component $\tilde{e}_{i j}$ and $h_{i j}^{k}$ are the assumed interpolation functions satisfying

$h_{i j}^{k}\left(r_{i j}^{l}, s_{i j}^{l}\right)=\delta_{k l}, \quad l=1, \ldots, n_{i j}$.

Note that this tying procedure is carried out on the elemental level for each individual element. Expressing the displacement-based covariant strain components in terms of the nodal displacements and rotations

$e_{i j}=\mathbf{B}_{i j} \mathbf{U}$,

where $\mathbf{B}$ is the strain-displacement matrix and $\mathbf{U}$ is the nodal displacement/rotation vector, we obtain

$\tilde{e}_{i j}=\left[\left.\sum_{k=1}^{n_{i j}} h_{i j}^{k}(r, s) \mathbf{B}_{i j}\right|_{\left(r_{i j}^{k}, k_{i j}^{k}, t\right)}\right] \mathbf{U}=\widetilde{\mathbf{B}}_{i j} \mathbf{U}$.

Then using the proper stress-strain matrix, the element stiffness matrix is constructed in the same manner as for the displacement-based element.

\section{Strain interpolation technique for isotropic triangular shell elements}

Two important points of the successful MITC technique are to use appropriate assumed strain interpolations in Eq. (5) and to carefully choose the tying points. In recent research [21], it was observed that a seemingly small change in the tying positions can result in significant differences in the predictive capability of the MITC9 shell element.

While the interpolation of the covariant strain components is quite easily achieved for quadrilateral elements, the interpolation is more difficult for triangular elements due to their shape and coordinate system. In this section we provide a systematic way to interpolate the strain components to reach isotropic MITC triangular shell elements.

\subsection{Strain interpolation methods}

Let us consider a three node isoparametric beam element. As shown in Fig. 1(a), the displacement-based element has a quadratic variation of transverse shear strain. In order to remove shear locking, we need to linearly interpolate the transverse shear strain in the beam element [1,2]. The linear transverse shear strain field can be determined by the two transverse shear strains sampled at two different tying points $\left(r=r_{1}\right.$ and $r=r_{2}=-r_{1}$ ). Three kind of approaches shown in Fig. 1(b)-(d) can be employed to determine the interpolation.

\section{Method- $i$}

Since we know that the resulting polynomial for $\tilde{e}_{r t}$ is linear, we assume

$\tilde{e}_{r t}=a+b r$.

Using the two conditions

$\tilde{e}_{r t}\left(r_{1}\right)=e_{r t}^{(1)}$,
$\tilde{e}_{r t}\left(r_{2}\right)=e_{r t}^{(2)}$, 
(a)

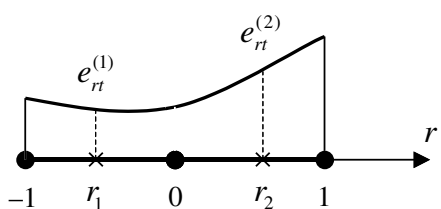

(c)

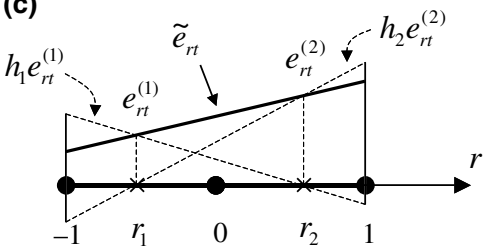

(b)

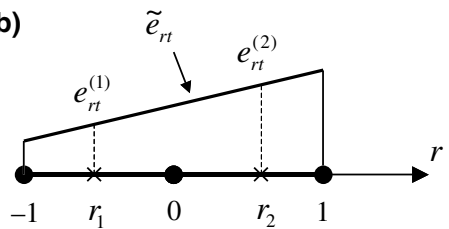

(d)

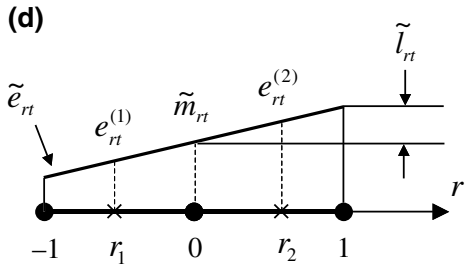

Fig. 1. Derivation of the interpolation functions from given tying points.

the unique pair of the coefficients, $a$ and $b$, can be determined, see Fig. 1(b).

\section{Method-ii}

In this method, shown in Fig. 1(c), we use the shape functions of the standard isoparametric procedure

$\tilde{e}_{r t}=\sum_{i=1}^{2} h_{i} e_{r t}^{(i)}=h_{1} e_{r t}^{(1)}+h_{2} e_{r t}^{(2)}$,

where $h_{1}$ and $h_{2}$ are the linear functions satisfying

$h_{i}\left(r_{j}\right)=\delta_{i j}$

Therefore, if we assume

$h_{1}=a_{1}+b_{1} r, \quad h_{2}=a_{2}+b_{2} r$

with four conditions

$h_{1}\left(r_{1}\right)=1, \quad h_{1}\left(r_{2}\right)=0$,

$h_{2}\left(r_{1}\right)=0, \quad h_{2}\left(r_{2}\right)=1$,

we can obtain the four coefficients $\left(a_{1}, b_{1}, a_{2}, b_{2}\right)$.

\section{New method}

We here propose a simple new method, described in Fig. 1(d). Since the order of the transverse shear strain of the displacement-based three node isoparametric beam element is quadratic, we start from

$\tilde{e}_{r t}=a+b r+c r^{2}$.

The following three conditions (imposing a linear variation) given at the nodes can be applied to evaluate $a, b$ and $c$ : $\tilde{e}_{r t}(-1)=\tilde{m}_{r t}-\tilde{l}_{r t}$,

$\tilde{e}_{r t}(0)=\tilde{m}_{r t}$,

$\tilde{e}_{r t}(1)=\tilde{m}_{r t}+\tilde{l}_{r t}$

where $\tilde{m}_{r t}$ is the mean value of the two tying strains and $\tilde{l}_{r t}$ is the difference between the value at the center $(r=0)$ and the edge $(r=1)$, that is,

$\tilde{m}_{r t}=\frac{1}{2}\left(e_{r t}^{(1)}+e_{r t}^{(2)}\right), \quad \tilde{l}_{r t}=\frac{e_{r t}^{(2)}-e_{r t}^{(1)}}{r_{2}-r_{1}}$.

Solving Eq. (16), we obtain

$\tilde{e}_{r t}=\tilde{m}_{r t}+\tilde{l}_{r t} r$.

Here, the coefficient of the second-order term, $c$, automatically vanishes. Note that while we tie the strains at $r=r_{1}$ and $r=r_{2}$, Eq. (16) considers the strains at nontying positions.

The three methods give exactly the same interpolation for this example. To use "method-i" and "method-ii", the interpolations start from linear polynomials, while, in the new method, the interpolation starts from the quadratic polynomial and the coefficient of the quadratic term automatically vanishes by imposition of the linear variation. Due to this property, the method can be used even when the exact space of functions for a $2 \mathrm{D}$ or $3 \mathrm{D}$ element is not known (see sections below).

Note that, for the example considered here, two unknown coefficients and two linear equations are considered by "method-i", four unknown coefficients and four linear equations are considered by "method-ii" and three unknown coefficients and three linear equations are considered by the proposed method.

The proposed method is powerful, specifically when we construct the transverse shear strain fields for isotropic MITC triangular shell elements. 


\subsection{Interpolation of transverse shear strain field}

We have two independent covariant transverse shear strains from which the complete transverse shear strain field of the element can be determined.

To construct isotropic transverse shear strain fields for MITC quadrilateral shell elements, we can separately interpolate the two transverse shear strains $\left(e_{r t}\right.$ and $\left.e_{s t}\right)$ corresponding to the two directions $r$ and $s$. Namely, in the natural coordinate system, each edge of a quadrilateral element is parallel to the corresponding opposite edge. Then, the elements automatically have isotropic transverse shear strain fields and behave isotropically.

However, to obtain isotropic transverse shear strain fields for an MITC triangular element, we need to have that the strain variations corresponding to the three edge directions of the element are identical. The main obstacle then comes from the fact that, although there are only two independent transverse shear strains $e_{r t}$ and $e_{s t}$, the additional transverse shear strain $e_{q t}$ corresponding to the hypotenuse of the right-angled triangle, see Fig. 2, in the natural coordinate system must be considered.

Fig. 2 shows how to find the transverse shear strain $e_{q t}$ from $e_{r t}$ and $e_{s t}$ at the point considered in the triangular element. The shear strain $e_{q t}$ is given by the tensor transformation

$e_{q t}=\frac{1}{\sqrt{2}}\left(e_{s t}-e_{r t}\right)$.

The first step is to choose the polynomial space of the assumed transverse shear strains, $\tilde{e}_{r t}$ and $\tilde{e}_{s t}$. The assumed transverse shear strain $\tilde{e}_{q t}$ is immediately given from Eq. (19). The following equations express this step:

$$
\begin{aligned}
\tilde{e}_{r t}= & a_{1}+b_{1} r+c_{1} s \ldots, \\
\tilde{e}_{s t}= & a_{2}+b_{2} r+c_{2} s \ldots, \\
\tilde{e}_{q t}= & \frac{1}{\sqrt{2}}\left(\tilde{e}_{s t}-\tilde{e}_{r t}\right)=\frac{1}{\sqrt{2}}\left\{\left(a_{2}+b_{2} r+c_{2} s \ldots\right)\right. \\
& \left.-\left(a_{1}+b_{1} r+c_{1} s \ldots\right)\right\},
\end{aligned}
$$

where the $a_{1}, b_{1}, \ldots$ and $a_{2}, b_{2}, \ldots$ denote the unknown coefficients of the strain interpolation polynomials.
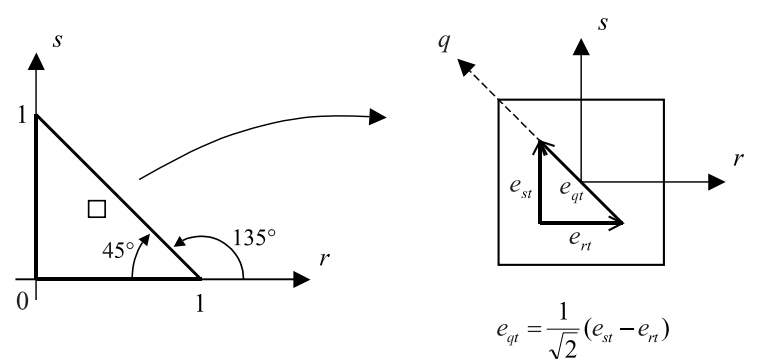

Fig. 2. Calculation of the transverse shear strain $e_{q t}$.

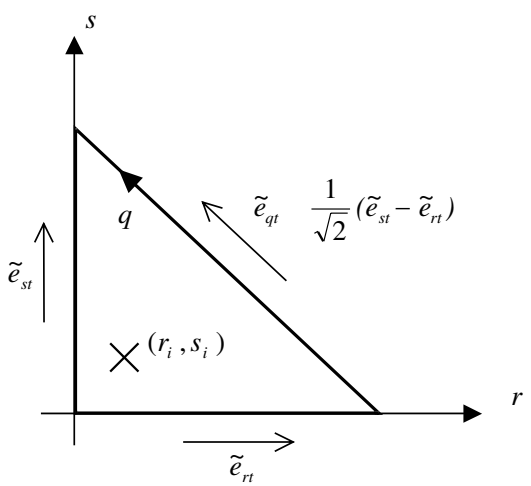

Fig. 3. Transverse shear strain relation and typical point used in strain evaluations.

The second step is to choose the strain tying positions. These positions should be located isotropically in the element. The displacement-based strains at these positions are tied to the assumed strain variations. This tying is achieved by evaluating the assumed strains at judiciously chosen points $\left(r_{i}, s_{i}\right), i=1,2, \ldots$ from the displacement-based strains, see Fig. 3,

$$
\begin{aligned}
& \tilde{e}_{r t}\left(r_{i}, s_{i}\right)=\ldots, \\
& \tilde{e}_{s t}\left(r_{i}, s_{i}\right)=\ldots, \\
& \tilde{e}_{q t}\left(r_{i}, s_{i}\right)=\ldots
\end{aligned}
$$

Note that as in Eq. (16) these points do not need to be the tying points.

The last step is to solve the resulting linear equations for the unknown coefficients in the assumed strain variations. The number of linearly independent equations should be equal to the number of unknowns.

This simple systematic procedure ensures the construction of isotropic transverse shear strain fields for MITC triangular shell elements. Here we discussed the construction of the isotropic transverse shear strain field by the new method proposed in the previous section. Note that "method-i" and "method-ii" are not generally applicable.

Of course, we should note that the assumed strain variations should be of lower order than the strains obtained by the assumed displacements.

To exemplify the procedure, consider a 3-node triangular shell element with constant transverse shear strain along its edges, see Fig. 4. The tying points are chosen at the center of the edges.

Step 1. The interpolation starts from

$$
\begin{aligned}
\tilde{e}_{r t} & =a_{1}+b_{1} r+c_{1} s \\
\tilde{e}_{s t} & =a_{2}+b_{2} r+c_{2} s \\
\tilde{e}_{q t} & =\frac{1}{\sqrt{2}}\left(\tilde{e}_{s t}-\tilde{e}_{r t}\right) \\
& =\frac{1}{\sqrt{2}}\left\{\left(a_{2}+b_{2} r+c_{2} s\right)-\left(a_{1}+b_{1} r+c_{1} s\right)\right\} .
\end{aligned}
$$



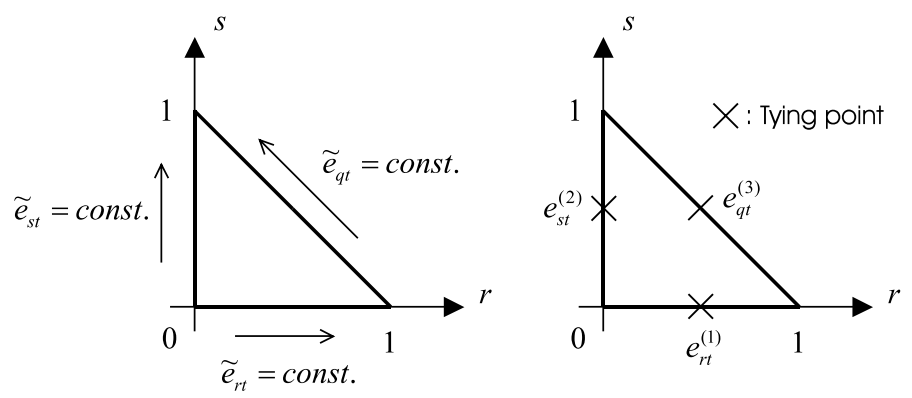

Fig. 4. Transverse shear strain tying positions for the 3-node triangular shell element with the constant transverse shear strain along its edges.

Step 2. The conditions are

$$
\begin{aligned}
& \tilde{e}_{r t}(0,0)=e_{r t}^{(1)}, \quad \tilde{e}_{r t}(1,0)=e_{r t}^{(1)}, \\
& \tilde{e}_{s t}(0,0)=e_{s t}^{(2)}, \quad \tilde{e}_{s t}(0,1)=e_{s t}^{(2)}, \\
& \tilde{e}_{q t}(1,0)=e_{q t}^{(3)}=\frac{1}{\sqrt{2}}\left(e_{s t}^{(3)}-e_{r t}^{(3)}\right), \\
& \tilde{e}_{q t}(0,1)=e_{q t}^{(3)}=\frac{1}{\sqrt{2}}\left(e_{s t}^{(3)}-e_{r t}^{(3)}\right)
\end{aligned}
$$

and we obtain six linearly independent equations for the unknown coefficients $a_{1}, b_{1}, \ldots, c_{2}$.

Step 3. We solve the linear equations and obtain

$$
\begin{array}{ll}
a_{1}=e_{r t}^{(1)}, & b_{1}=0, \quad c_{1}=e_{s t}^{(2)}-e_{r t}^{(1)}-e_{s t}^{(3)}+e_{r t}^{(3)}, \\
a_{2}=e_{s t}^{(2)}, & b_{2}=-c_{1}, \quad c_{2}=0,
\end{array}
$$

which gives the isotropic transverse shear strain field

$$
\begin{aligned}
& \tilde{e}_{r t}=e_{r t}^{(1)}+c s, \\
& \tilde{e}_{s t}=e_{s t}^{(2)}-c r,
\end{aligned}
$$

where $c=e_{s t}^{(2)}-e_{r t}^{(1)}-e_{s t}^{(3)}+e_{r t}^{(3)}$.

\subsection{Interpolation of in-plane strain field}

In the formulation of the quadrilateral MITC shell elements, the covariant in-plane strains are independently treated in a straight-forward manner. However, to reach MITC isotropic triangular elements, additional considerations arise.

The interpolation of the in-plane strain field starts from well known basic facts of mechanics. The complete in-plane strain field is usually given by three strains, that is, two normal strains (usually, $e_{r r}$ and $e_{s s}$ ) and one inplane shear strain $\left(e_{r s}\right)$. However, three independent normal strains can also give the complete in-plane strain field.

To construct the isotropic in-plane strain field, we introduce the normal strain, $e_{q q}$, in the hypotenuse direction of the right-angled triangle in the natural coordinate system

$e_{q q}=\frac{e_{r r}+e_{s s}}{2}+\frac{e_{r r}-e_{s s}}{2} \cos (2 \theta)+e_{r s} \sin (2 \theta)$

with $\theta=135^{\circ}(\cos (2 \theta)=0$ and $\sin (2 \theta)=-1)$, see Figs. 2 and 5.

The first step for the construction of the isotropic inplane strain field is to independently interpolate the three in-plane strains $e_{r r}, e_{s s}$ and $e_{q q}$ with the same order of polynomials

$\tilde{e}_{r r}=a_{1}+b_{1} r+c_{1} s \ldots$,
$\tilde{e}_{s s}=a_{2}+b_{2} r+c_{2} s \ldots$,
$\tilde{e}_{q q}=a_{3}+b_{3} r+c_{3} s \ldots$,

where $a_{1}, b_{1}, \ldots, a_{2}, b_{2}, \ldots$, and $a_{3}, b_{3}, \ldots$ denote the unknown coefficients of the strain polynomials. Of course, polynomials of lower order than implied by the assumed displacements should be used.

In the second step, we select isotropic tying positions in the triangular element and evaluate the assumed strains from the displacement-based strains at

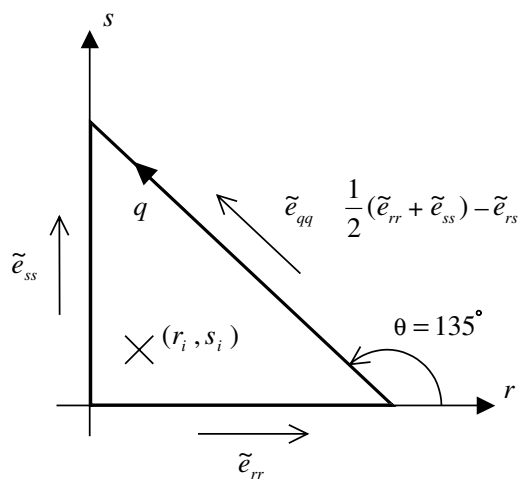

Fig. 5. In-plane strain relation and typical point used in strain evaluations. 
judiciously chosen points $\left(r_{i}, s_{i}\right)$ in the element, $i=1,2, \ldots$.

$$
\begin{aligned}
& \tilde{e}_{r r}\left(r_{i}, s_{i}\right)=\ldots, \\
& \tilde{e}_{s s}\left(r_{i}, s_{i}\right)=\ldots \\
& \tilde{e}_{q q}\left(r_{i}, s_{i}\right)=\ldots
\end{aligned}
$$

As for the transverse shear strain interpolations, the $\left(r_{i}, s_{i}\right)$ do not need to be tying positions. The number of linearly independent equations reached should be equal to the number of unknown coefficients in Eq. (27).

The last step is to solve the resulting linear equations for the unknown coefficients in Eq. (27). The strain $\tilde{e}_{r s}$, which is needed for the finite element formulation, is directly obtained as

$\tilde{e}_{r s}(r, s)=\frac{1}{2}\left\{\tilde{e}_{r r}(r, s)+\tilde{e}_{s s}(r, s)\right\}-\tilde{e}_{q q}(r, s)$.

As an example, consider a 6-node triangular shell element with linear normal strain variations along its edges. Fig. 6 shows the tying points corresponding to each normal strain. Note that this tying is just one of the possible schemes for triangular shell elements with linearly varying normal strains along the edges, that is, various tying schemes could be used.
Step 1 . We assume the starting polynomials

$$
\begin{aligned}
& \tilde{e}_{r r}=a_{1}+b_{1} r+c_{1} s, \\
& \tilde{e}_{s s}=a_{2}+b_{2} r+c_{2} s, \\
& \tilde{e}_{q q}=a_{3}+b_{3} r+c_{3}(1-r-s),
\end{aligned}
$$

where, of course, $\tilde{e}_{q q}=a_{3}+b_{3} r+c_{3} s$ can be used instead of $\tilde{e}_{q q}=a_{3}+b_{3} r+c_{3}(1-r-s)$.

Step 2. The conditions used are

$$
\begin{aligned}
& \tilde{e}_{r r}(0,0)=\tilde{m}_{r r}^{(1)}-\tilde{l}_{r r}^{(1)}, \quad \tilde{e}_{r r}(1 / 2,0)=\tilde{m}_{r r}^{(1)}, \\
& \tilde{e}_{r r}(1,0)=\tilde{m}_{r r}^{(1)}+\tilde{l}_{r r}^{(1)}, \quad \tilde{e}_{s s}(0,0)=\tilde{m}_{s s}^{(2)}-\tilde{l}_{s s}^{(2)}, \\
& \tilde{e}_{s s}(0,1 / 2)=\tilde{m}_{s s}^{(2)}, \quad \tilde{e}_{s s}(0,1)=\tilde{m}_{s s}^{(2)}+\tilde{l}_{s s}^{(2)} \\
& \tilde{e}_{q q}(1,0)=\tilde{m}_{q q}^{(3)}-\tilde{l}_{q q}^{(3)}, \quad \tilde{e}_{q q}(1 / 2,1 / 2)=\tilde{m}_{q q}^{(3)}, \\
& \tilde{e}_{q q}(0,1)=\tilde{m}_{q q}^{(3)}+\tilde{l}_{q q}^{(3)}, \quad \tilde{e}_{r r}\left(r_{1}, 1 / \sqrt{3}\right)=e_{c r}^{(1)}, \\
& \tilde{e}_{s s}\left(1 / \sqrt{3}, s_{1}\right)=e_{c s s}^{(2)}, \quad \tilde{e}_{q q}\left(r_{1}, s_{1}\right)=e_{c q q}^{(3)}
\end{aligned}
$$

where

$$
\begin{aligned}
& \tilde{m}_{j j}^{(i)}=\frac{1}{2}\left(e_{1 j j}^{(i)}+e_{2 j j}^{(i)}\right), \quad \tilde{l}_{j j}^{(i)}=\frac{\sqrt{3}}{2}\left(e_{2 j j}^{(i)}-e_{1 j j}^{(i)}\right) \\
& \text { with } j=r, s, q \quad \text { for } \quad i=1,2,3
\end{aligned}
$$
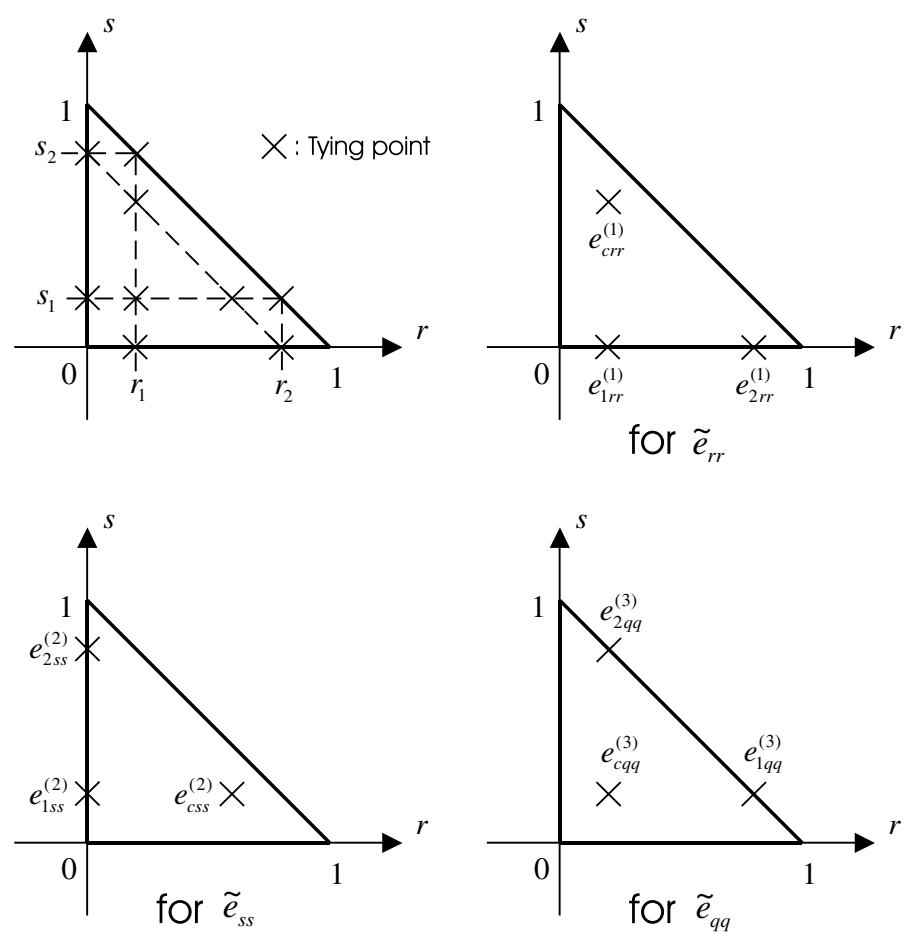

Fig. 6. Strain tying positions for the 6-node triangular shell element with linear normal strain along edges; $r_{1}=s_{1}=\frac{1}{2}-\frac{1}{2 \sqrt{3}}$ and $r_{2}=s_{2}=\frac{1}{2}+\frac{1}{2 \sqrt{3}}$. 
and

$r_{1}=s_{1}=\frac{1}{2}-\frac{1}{2 \sqrt{3}}, \quad r_{2}=s_{2}=\frac{1}{2}+\frac{1}{2 \sqrt{3}}$.

Step 3. The solution of the equations gives

$$
\begin{aligned}
& a_{1}=\tilde{m}_{r r}^{(1)}-\tilde{l}_{r r}^{(1)}, \quad b_{1}=2 \tilde{l}_{l r}^{(1)}, \\
& a_{2}=\tilde{m}_{s s}^{(2)}-\tilde{l}_{s s}^{(2)}, \quad c_{2}=2 \tilde{l}_{s s}^{(2)}, \\
& a_{3}=\tilde{m}_{q q}^{(3)}+\tilde{l}_{q q}^{(3)}, \quad b_{3}=-2 \tilde{l}_{q q}^{(3)}, \\
& c_{1}=\sqrt{3}\left(e_{c r}^{(1)}-a_{1}-b_{1} r_{1}\right), \\
& b_{2}=\sqrt{3}\left(e_{c s s}^{(2)}-a_{2}-c_{2} s_{1}\right), \\
& c_{3}=\sqrt{3}\left(e_{c q q}^{(3)}-a_{3}-b_{3} r_{1}\right) .
\end{aligned}
$$

As a result, we obtain the isotropic in-plane strain field and the interpolation function for the in-plane shear strain $\tilde{e}_{r s}$ is immediately given by Eq. (29).

\section{MITC isotropic triangular shell elements}

A successful MITC shell element is based on strain interpolations that result in good behavior in both bending and membrane dominated problems. However, in many cases, if the interpolation of assumed strains induces good behavior of the element in bending dominated shell problems, the element is too flexible (or unstable in the worst case) in membrane dominated shell problems. On the other hand, the element might be too stiff or lock in bending dominated problems.

Therefore, the success is in using well balanced strain interpolations in the formulation. When developing MITC triangular shell finite elements, the goal is to eliminate shear and membrane locking in bending dominated shell problems and to keep the consistency of the element in membrane dominated shell problems.

The optimal strain interpolations and tying scheme/ points for the MITC technique depend on the displacement interpolations used, that is, the polynomial space of the element displacement interpolation. In the previous section, we discussed how to obtain isotropic strain fields for the MITC technique. Given a displacement interpolation, there exist a number of possible interpolation schemes for the transverse shear strains and the in-plane strains. To construct the MITC triangular shell finite elements, each transverse shear strain interpolation scheme can be combined with various inplane strain interpolation schemes. As a result, we can develop many new shell finite elements, but only few elements will be effective for practical purposes and, of course, we are searching the "optimal" element for a given displacement interpolation.
Here, we focus on the behavior of relatively competitive elements among possible elements, which are proposed as follows:

\section{MITC3}

Since the geometry of the 3-node triangular shell element is always flat, we only use, like for the MITC4 element, the mixed interpolation for the transverse shear strains. We assume that the transverse shear strains of the element are constant along edges. The tying and interpolation schemes are shown in Fig. 4 and in Eq. (25), respectively.

\section{MITC6- $a$}

For this 6-node MITC triangular shell element, linear transverse shear strains along edges are assumed and therefore two tying points at each edge are chosen. We have one tying point $(r=1 / 3, s=1 / 3)$ to express quadratic variations of strains inside the element. Fig. 7(a) shows the tying positions for this scheme, in which

$$
\begin{aligned}
& r_{1}=s_{1}=\frac{1}{2}-\frac{1}{2 \sqrt{3}}, \quad r_{2}=s_{2}=\frac{1}{2}+\frac{1}{2 \sqrt{3}}, \\
& r_{3}=s_{3}=\frac{1}{3} .
\end{aligned}
$$

Note that, if we change the values of $r_{1}, s_{1}$ and $r_{2}, s_{2}$, the element will behave differently but a good predictive capability of the element is obtained with the values in Eq. (35).

We assume the strains to be given by

$$
\begin{aligned}
& \tilde{e}_{r t}=a_{1}+b_{1} r+c_{1} s+d_{1} r s+e_{1} r^{2}+f_{1} s^{2}, \\
& \tilde{e}_{s t}=a_{2}+b_{2} r+c_{2} s+d_{2} r s+e_{2} r^{2}+f_{2} s^{2}
\end{aligned}
$$

and have

$$
\begin{aligned}
a_{1}= & \tilde{m}_{r t}^{(1)}-\tilde{l}_{r t}^{(1)}, \quad b_{1}=2 \tilde{l}_{r t}^{(1)}, \quad e_{1}=0, \\
a_{2}= & \tilde{m}_{s t}^{(2)}-\tilde{l}_{s t}^{(2)}, \quad c_{2}=2 \tilde{l}_{s t}^{(2)}, \quad f_{2}=0, \\
c_{1}= & 6 e_{c r t}-3 e_{c s t}+2 \tilde{m}_{s t}^{(3)}-2 \tilde{m}_{r t}^{(3)}-4 a_{1}-b_{1}+a_{2}, \\
b_{2}= & -3 e_{c r t}+6 e_{c s t}-2 \tilde{m}_{s t}^{(3)}+2 \tilde{m}_{r t}^{(3)}+a_{1}-4 a_{2}-c_{2}, \\
e_{2}= & 3 e_{c r t}-6 e_{c s t}+3 \tilde{m}_{s t}^{(3)}-\tilde{l}_{s t}^{(3)}-3 \tilde{m}_{r t}^{(3)}+\tilde{l}_{r t}^{(3)} \\
& +b_{1}+3 a_{2}+c_{2}, \\
f_{1}= & -6 e_{c r t}+3 e_{c s t}-3 \tilde{m}_{s t}^{(3)}-\tilde{l}_{s t}^{(3)}+3 \tilde{m}_{r t}^{(3)} \\
& +\tilde{l}_{r t}^{(3)}+3 a_{1}+b_{1}+c_{2}, \\
d_{1}= & -e_{2}, \quad d_{2}=-f_{1},
\end{aligned}
$$



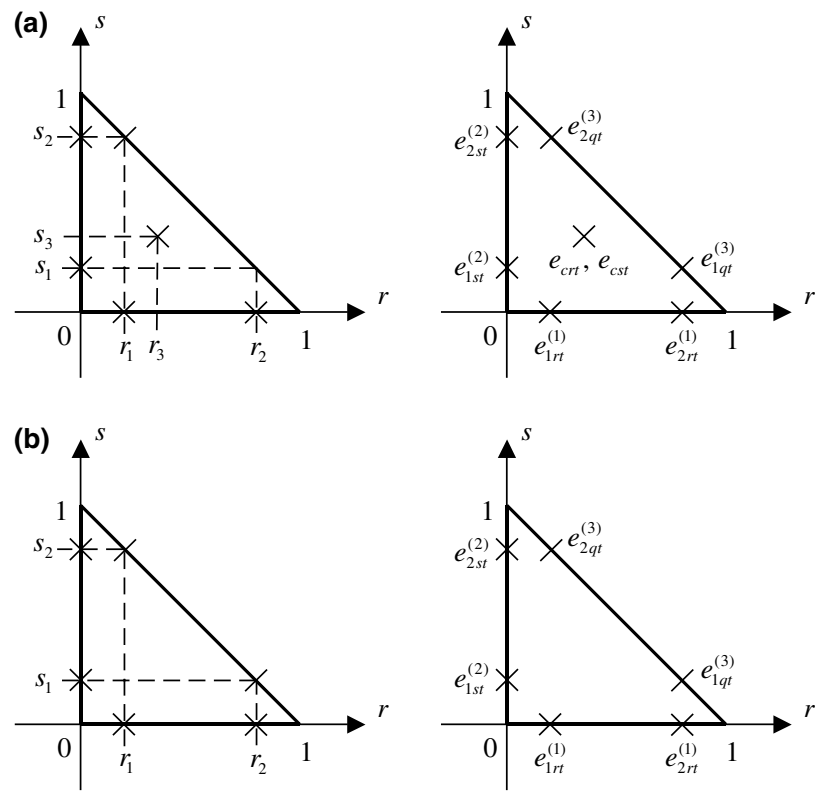

Fig. 7. Tying points for the transverse shear strain interpolation of the 6-node MITC triangular shell elements: (a) for MITC6-a and (b) for MITC6-b.

where

$\tilde{m}_{j t}^{(i)}=\frac{1}{2}\left(e_{1 j t}^{(i)}+e_{2 j t}^{(i)}\right), \quad \tilde{l}_{j t}^{(i)}=\frac{\sqrt{3}}{2}\left(e_{2 j t}^{(i)}-e_{1 j t}^{(i)}\right)$

with $j=r, s$ for $i=1,2,3$.

It is interesting to note that this scheme is very similar to the interpolation scheme of the MITC7 plate bending element in reference [9]. For this element similar tying points are used and the interpolation functions, which belong to a "rotated Raviart-Thomas space", are given as

$\tilde{e}_{r t}=a_{1}+b_{1} r+c_{1} s+s(d r+e s)$,

$\tilde{e}_{s t}=a_{2}+b_{2} r+c_{2} s-r(d r+e s)$.

We use the in-plane strain interpolation scheme given by Eqs. (30)-(34).

\section{MITC6-b}

Considering the transverse shear strains, this 6-node MITC triangular shell element has the same edge tying points as the MITC6-a element. Also "linear transverse shear strains along edges" are assumed. However, we do not have any internal tying point and the strain variations are linear inside the element. Fig. 7(b) shows the tying positions.

The transverse shear strain interpolations used are

$$
\begin{aligned}
& \tilde{e}_{r t}=a_{1}+b_{1} r+c_{1} s, \\
& \tilde{e}_{s t}=a_{2}+b_{2} r+c_{2} s,
\end{aligned}
$$

where using Eq. (38),

$$
\begin{aligned}
& a_{1}=\tilde{m}_{r t}^{(1)}-\tilde{l}_{r t}^{(1)}, \quad b_{1}=2 \tilde{l}_{r t}^{(1)}, \\
& a_{2}=\tilde{m}_{s t}^{(2)}-\tilde{l}_{s t}^{(2)}, \quad c_{2}=2 \tilde{l}_{s t}^{(2)}, \\
& c_{1}=\left(a_{2}+c_{2}-a_{1}\right)-\left(\tilde{m}_{s t}^{(3)}+\tilde{l}_{s t}^{(3)}-\tilde{m}_{r t}^{(3)}-\tilde{l}_{r t}^{(3)}\right), \\
& b_{2}=\left(a_{1}+b_{1}-a_{2}\right)+\left(\tilde{m}_{s t}^{(3)}-\tilde{l}_{s t}^{(3)}-\tilde{m}_{r t}^{(3)}+\tilde{l}_{r t}^{(3)}\right) .
\end{aligned}
$$

The same in-plane strain interpolation scheme as for the MITC6-a element is employed.

Fig. 8 summarizes the strain interpolation schemes of the selected MITC triangular shell elements. We may note the geometric relationships between the tying points used for the triangular MITC3 and MITC6 elements and the MITC4 [6] and MITC9 quadrilateral elements [21]. Fig. 9 shows these relationships.

\section{Numerical results}

In this section, we report upon various numerical tests of the MITC triangular shell finite elements, MITC3, MITC6-a and MITC6-b. Selected basic tests show whether the elements satisfy the minimum requirements, see Section 5.1. To investigate in detail the predictive capability of the proposed elements, we performed convergence studies for various shell problems [2], see Sections 5.2-5.4.

For convergence studies, we use the s-norm [14] defined as 


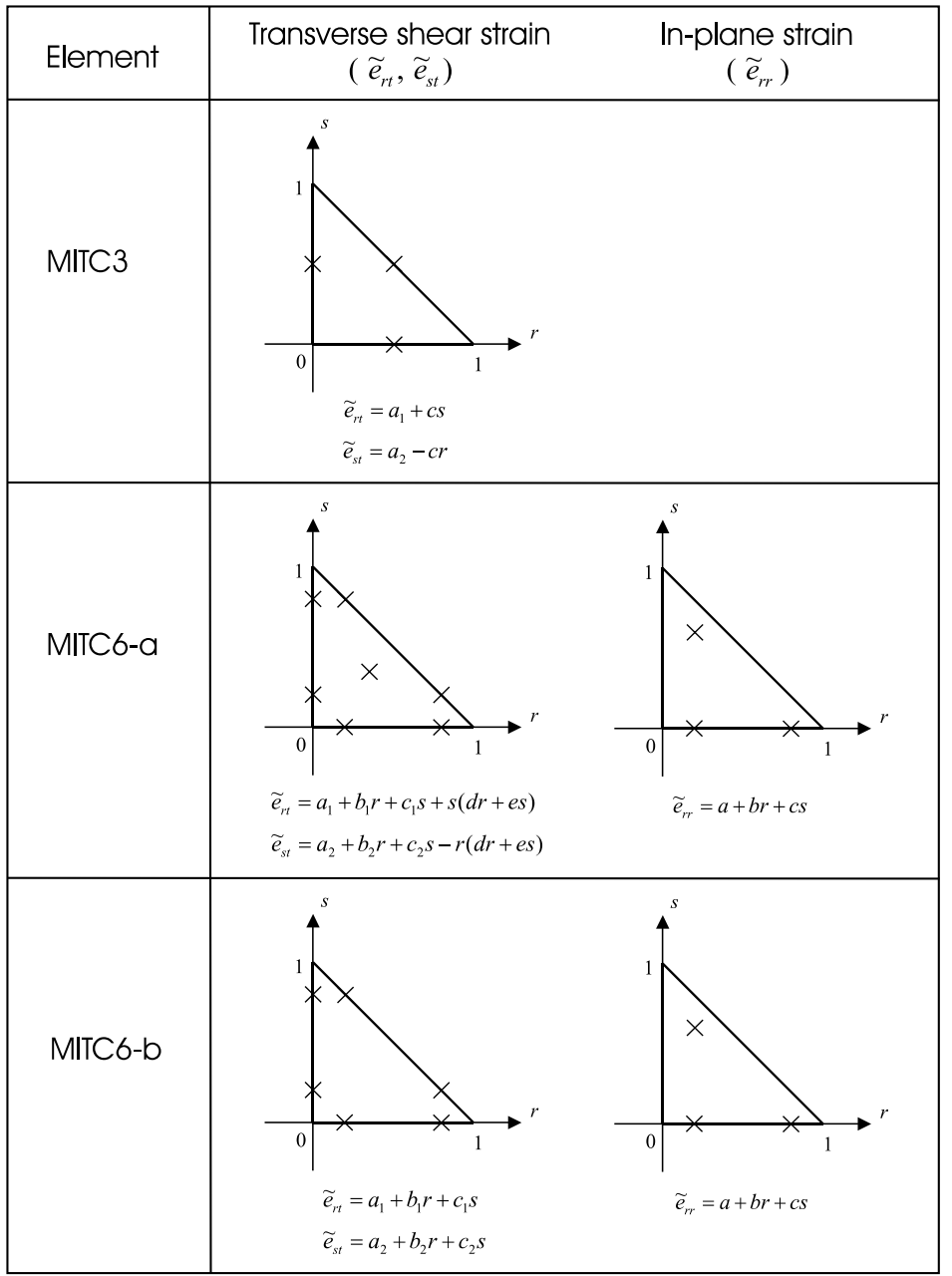

Fig. 8. Strain interpolation schemes and tying points of the MITC triangular shell finite elements.

$\left\|\vec{u}-\vec{u}_{h}\right\|_{s}^{2}=\int_{\Omega} \Delta \vec{\epsilon}^{T} \Delta \vec{\sigma} \mathrm{d} \Omega$,

where $\vec{u}$ denotes the exact solution and $\vec{u}_{h}$ denotes the solution of the finite element discretization. Here, $\vec{\epsilon}$ and $\vec{\sigma}$ are the strain vector and the stress vector in the global Cartesian coordinate system, respectively, defined by

$\vec{\epsilon}=\left[\epsilon_{x x}, \epsilon_{y y}, \epsilon_{z z}, 2 \epsilon_{x y}, 2 \epsilon_{y z}, 2 \epsilon_{z x}\right]^{\mathrm{T}}$,

$\vec{\sigma}=\left[\sigma_{x x}, \sigma_{y y}, \sigma_{z z}, \sigma_{x y}, \sigma_{y z}, \sigma_{z x}\right]^{\mathrm{T}}$

and

$$
\begin{aligned}
& \Delta \vec{\epsilon}=\vec{\epsilon}-\vec{\epsilon}_{h}=\mathbf{B}(\vec{x}) \mathbf{U}-\mathbf{B}_{h}\left(\vec{x}_{h}\right) \mathbf{U}_{h}, \\
& \Delta \vec{\sigma}=\vec{\sigma}-\vec{\sigma}_{h}=\mathbf{C}(\vec{x}) \mathbf{B}(\vec{x}) \mathbf{U}-\mathbf{C}_{h}\left(\vec{x}_{h}\right) \mathbf{B}_{h}\left(\vec{x}_{h}\right) \mathbf{U}_{h},
\end{aligned}
$$

where $\mathbf{C}$ denotes the material stress-strain matrix and $\mathbf{B}$ is the strain-displacement operator. The position vectors $\vec{x}$ and $\vec{x}_{h}$ correspond to the continuum domain and the discretized domain, respectively, and the relationship between them is

$\vec{x}=\Pi\left(\vec{x}_{h}\right)$,

where $\Pi$ defines a one-to-one mapping.

In the practical use of this norm, the finite element solution using a very fine mesh is adopted instead of the exact solution. Using the reference solution, the $s$-norm in Eq. (42) can be approximated by

$$
\left\|\vec{u}_{\mathrm{ref}}-\vec{u}_{h}\right\|_{s}^{2}=\int_{\Omega_{\mathrm{ref}}} \Delta \vec{\epsilon}^{T} \Delta \vec{\sigma} \mathrm{d} \Omega_{\mathrm{ref}}
$$

with

$$
\begin{aligned}
\Delta \vec{\epsilon}=\vec{\epsilon}_{\text {ref }}-\vec{\epsilon}_{h}= & \mathbf{B}_{\text {ref }}(\vec{x}) \mathbf{U}_{\text {ref }}-\mathbf{B}_{h}\left(\vec{x}_{h}\right) \mathbf{U}_{h}, \\
\Delta \vec{\sigma}=\vec{\sigma}_{\text {ref }}-\vec{\sigma}_{h}= & \mathbf{C}_{\text {ref }}(\vec{x}) \mathbf{B}_{\text {ref }}(\vec{x}) \mathbf{U}_{\text {ref }} \\
& -\mathbf{C}_{h}\left(\vec{x}_{h}\right) \mathbf{B}_{h}\left(\vec{x}_{h}\right) \mathbf{U}_{h} .
\end{aligned}
$$


(a)

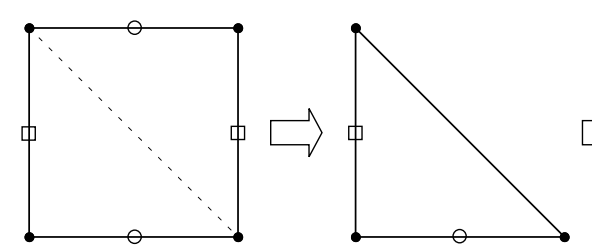

MITC4

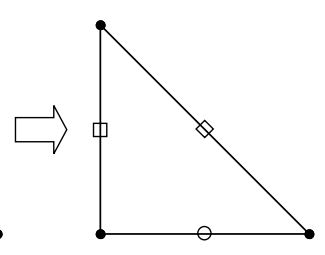

MITC3

(b)
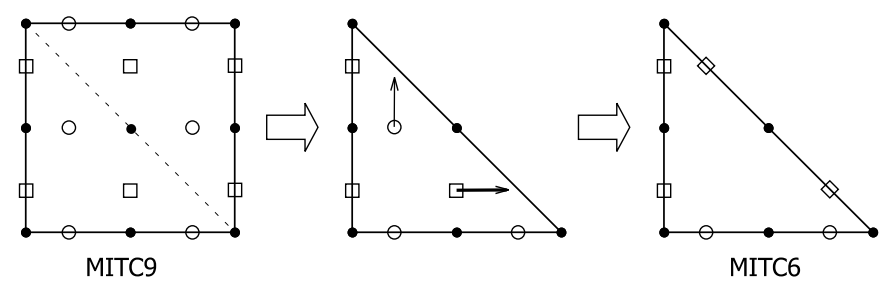

(c)
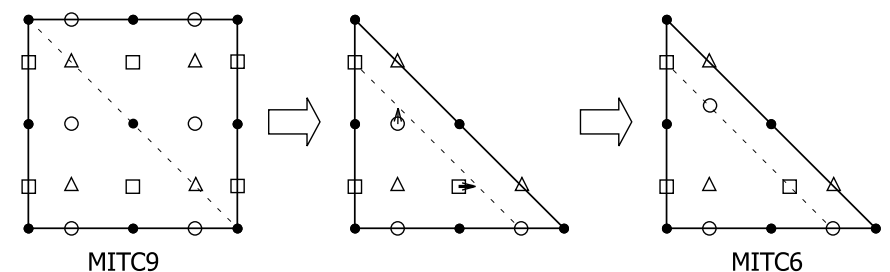

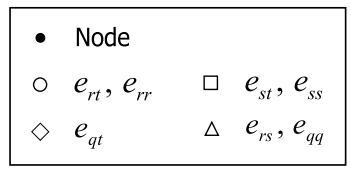

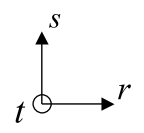

Fig. 9. Geometric relationships between tying points used for triangular and quadrilateral MITC shell elements. For the MITC9 element, see Ref. [21]: (a) selection of tying points for the transverse shear strains of the MITC3 element; (b) selection of edge tying points for the transverse shear strains of the MITC6 element and (c) selection of tying points for the in-plane strains of the MITC6 element.

To consider the convergence of the discretization schemes with various thicknesses, we use the relative error given by

relative error $=\frac{\left\|\vec{u}_{\text {ref }}-\vec{u}_{h}\right\|_{s}^{2}}{\left\|\vec{u}_{\mathrm{ref}}\right\|_{s}^{2}}$.

\subsection{Basic tests}

The following basic tests are performed as basic requirements for the triangular shell elements.

- Isotropic element test. Although the theory shows that the proposed elements are isotropic, we include this numerical test to illustrate the isotropy that triangular elements, in general, should satisfy. Considering any geometrical triangular element, this test should be passed. The test is performed by analyzing the three same triangular elements with different no- dal numbering sequences as shown in Fig. 10. The $r$ axis and $s$-axis always run from nodes 1 to 2 and nodes 1 to 3 , respectively. To pass the test, exactly the same response should be obtained for all possible (three-dimensional) tip forces and moments.

- Zero energy mode test. This test is performed by counting the number of zero eigenvalues of the stiffness matrix of one unsupported shell finite element, which should be exactly six, and the corresponding eigenvectors should of course be physical rigid body modes. We recommend that, when performing this test, various possible geometries be taken because an element might pass the test for a certain geometry but not for other geometries.

- Patch test. The patch test has been widely used to test elements, despite its limitations for mixed formulations, see Ref. [1]. We use the test here in numerical form to merely assess the sensitivity of our elements to geometric distortions. The mesh used for the patch test is taken from Ref. [1] and shown in Fig. 11. The 

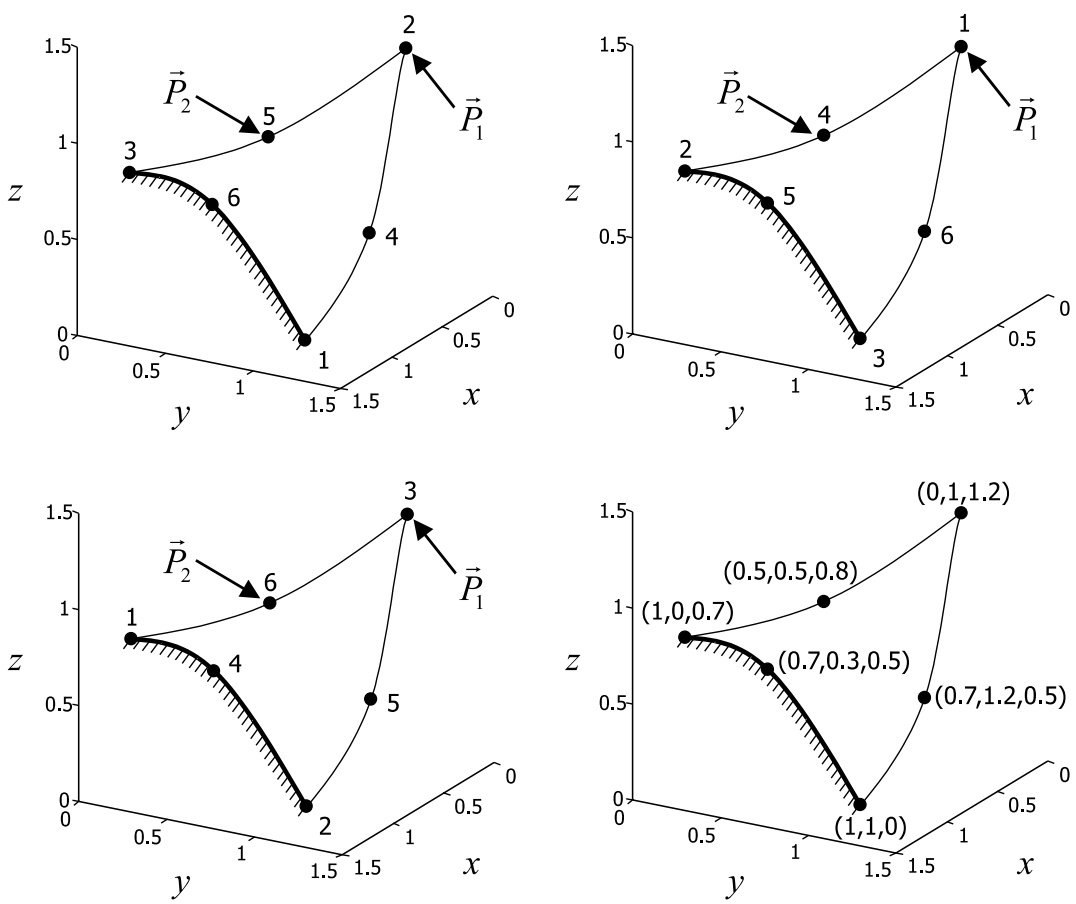

Fig. 10. Isotropic element test of the 6-node triangular shell element. $\vec{P}_{i}=\left\{f_{x}, f_{y}, f_{z}, m_{\alpha}, m_{\beta}\right\}^{\mathrm{T}}$.

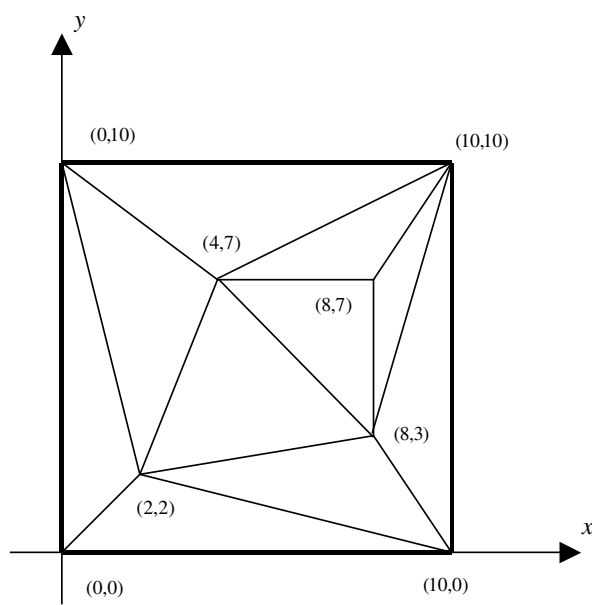

Fig. 11. Mesh used for the patch tests.

minimum number of degrees of freedom is constrained to prevent rigid body motion and the nodal point forces which should result in constant stress conditions are applied. The patch test is passed if indeed constant stress fields are calculated.

The results of the basic tests are reported in Table 1 . We notice that all elements proposed here pass all basic tests.

\subsection{Clamped plate problem}

We consider the plate bending problem shown in Fig. 12. The square plate of dimension $2 L \times 2 L$ with uniform thickness is subjected to a uniform pressure normal to the flat surface and all edges are fully clamped. Due to symmetry, only one quarter model is considered (region ABCD shown in Fig. 12) with the following symmetry and boundary conditions imposed:

$u_{x}=\theta_{y}=0 \quad$ along $\mathrm{BC}$,

$u_{y}=\theta_{x}=0 \quad$ along DC, and

$u_{x}=u_{y}=u_{z}=\theta_{x}=\theta_{y}=0$ along $\mathrm{AB}$ and $\mathrm{AD}$.

Table 1

Basic test results of the MITC triangular shell finite elements

\begin{tabular}{lllll}
\hline Element & Isotropic element test & Zero energy mode test & Membrane patch test & Bending patch test \\
\hline MITC3 & Pass & Pass & Pass & Pass \\
MITC6-a & Pass & Pass & Pass & Pass \\
MITC6-b & Pass & Pass & Pass & Pass \\
\hline
\end{tabular}




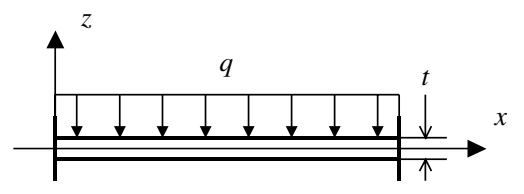

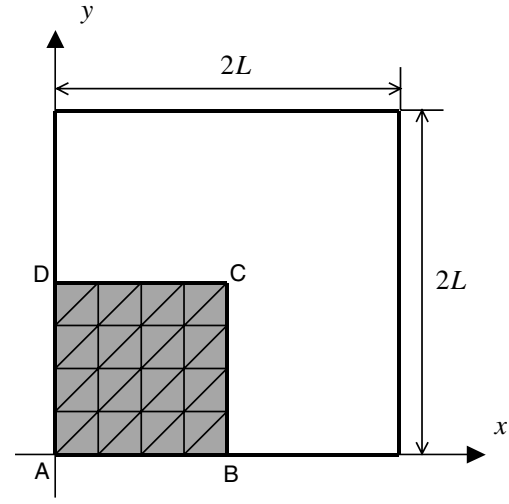

(a)

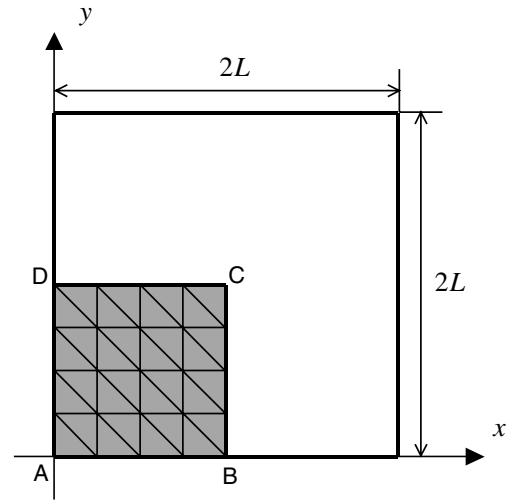

(b)

Fig. 12. Clamped plate under uniform pressure load with uniform $4 \times 4$ meshes of triangular elements $\left(L=1.0, E=1.7472 \times 10^{7}\right.$ and $v=0.3)$.
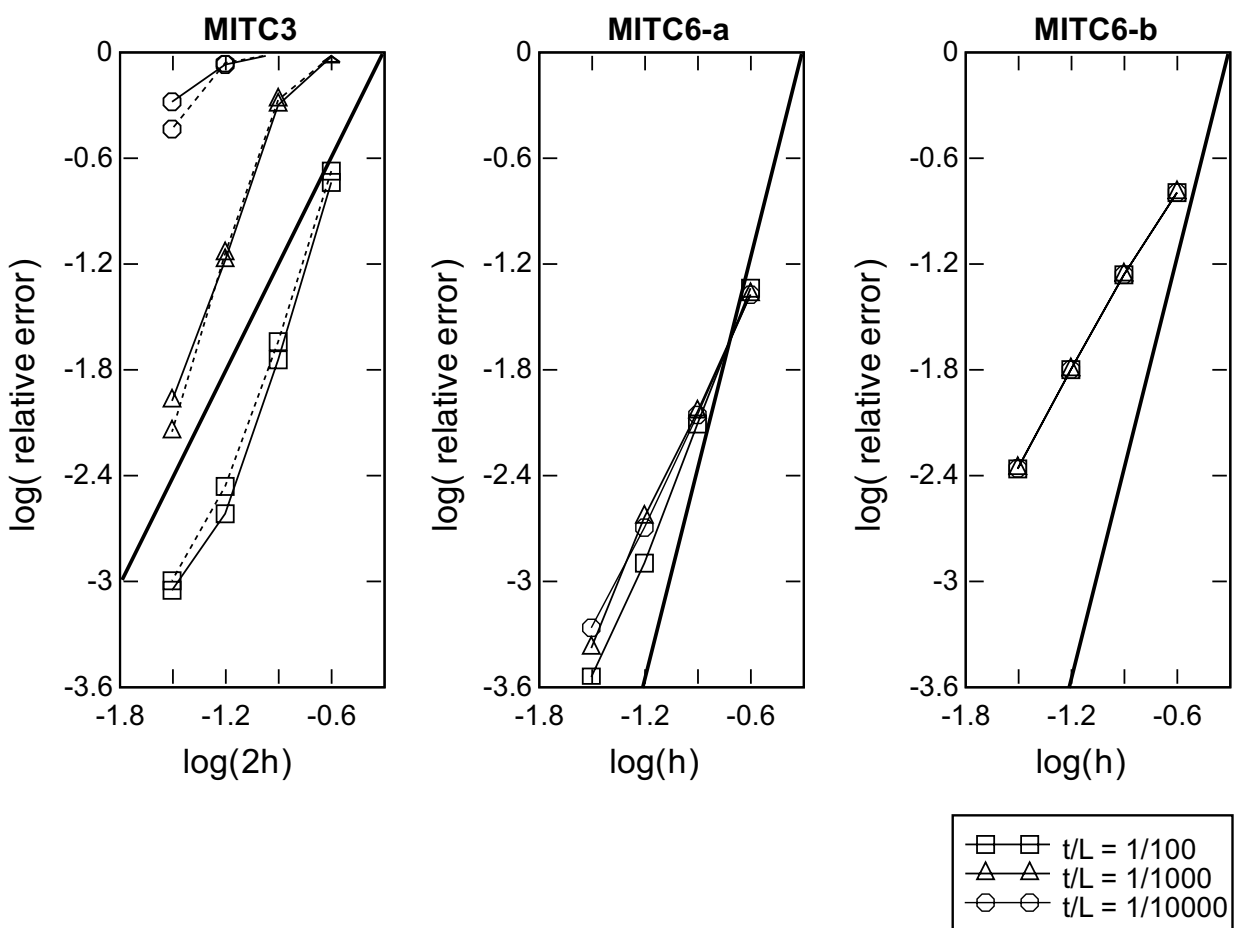

Fig. 13. Convergence curves for the clamped plate problem. The bold line shows the optimal convergence rate, which is 2.0 for linear elements and 4.0 for quadratic elements. For the MITC3 element, the solid and dotted lines correspond, respectively, to the results obtained using the meshes in Fig. 12(a) (solid line results) and (b) (dotted line results).

Fig. 13 reports the convergence of the MITC triangular shell elements in the relative error of Eq. (48). We use the solution using the MITC9 element with a mesh of $96 \times 96$ elements as reference. As the plate thickness 
decreases, the MITC3 element locks but still good accuracy characteristics are seen for $t / L$ up to about 1/1000. The MITC6-a element shows almost optimal convergence.

\subsection{Cylindrical shell problems}

We consider a cylindrical shell of uniform thickness $t$, length $2 L$ and radius $R$, see Fig. 14 . The shell is loaded by the pressure distribution $p(\theta)$ normal to the shell surface,

$p(\theta)=p_{0} \cos (2 \theta)$.

This shell shows two different asymptotic behaviors depending on the boundary conditions at its ends: bending dominated behavior when the ends are free and membrane dominated behavior when the ends are clamped.

By symmetry, we can limit calculations to the region $\mathrm{ABCD}$. For the free edge case, the following boundary conditions are imposed:
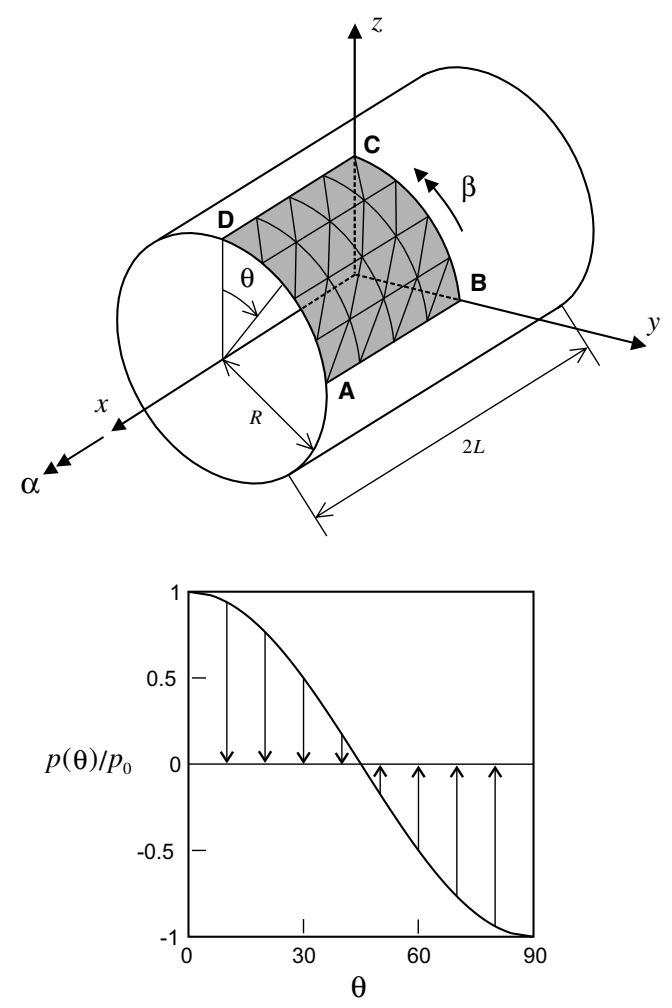

Fig. 14. Cylindrical shell problem with a $4 \times 4$ mesh of triangular elements $\left(L=R=1.0, \quad E=2.0 \times 10^{5}, \quad v=1 / 3\right.$ and $\left.p_{0}=1.0\right)$. $u_{x}=\beta=0$ along BC,

$u_{y}=\alpha=0$ along DC, and

$u_{z}=\alpha=0$ along AB.

For the clamped case, the boundary conditions are

$u_{x}=\beta=0$ along $\mathrm{BC}$,

$u_{y}=\alpha=0$ along DC,

$u_{z}=\alpha=0$ along $\mathrm{AB}$, and

$u_{x}=u_{y}=u_{z}=\alpha=\beta=0$ along AD.

A detailed study of this shell problem is presented in Ref. [12]. The relative error used here is based on the reference solution obtained with a mesh of $96 \times 96$ MITC9 shell elements.

Fig. 15 displays the convergence curves of the triangular shell elements for the clamped case. We note that the MITC3, MITC6-a and MITC6-b elements show good convergence behavior.

Fig. 16 presents the convergence curves for the free case. The MITC6-a element shows here as well good convergence.

\subsection{Hyperboloid shell problems}

The following two test problems use the same geometry given in Fig. 17 and the same loading. The midsurface of this shell structure is described by [2]

$x^{2}+z^{2}=1+y^{2} ; \quad y \in[-1,1]$.

The loading imposed is the smoothly varying periodic pressure normal to the surface,

$p(\theta)=p_{0} \cos (2 \theta)$,

which is the same distribution as shown in Fig. 14.

A bending dominated problem is obtained when both ends are free and a membrane dominated problem is obtained when the ends are clamped.

Using symmetry, the analyses are performed using one eighth of the structure, the shaded region $\mathrm{ABCD}$ in Fig. 17(a). Considering the boundary conditions, we have for the free case

$u_{z}=\beta=0$ along $\mathrm{BC}$,

$u_{x}=\beta=0$ along $\mathrm{AD}, \quad$ and

$u_{y}=\alpha=0$ along DC

and, for the clamped case

$u_{z}=\beta=0$ along BC,

$u_{x}=\beta=0$ along $\mathrm{AD}$,

$u_{y}=\alpha=0$ along DC, and

$u_{x}=u_{y}=u_{z}=\alpha=\beta=0$ along $\mathrm{AB}$. 

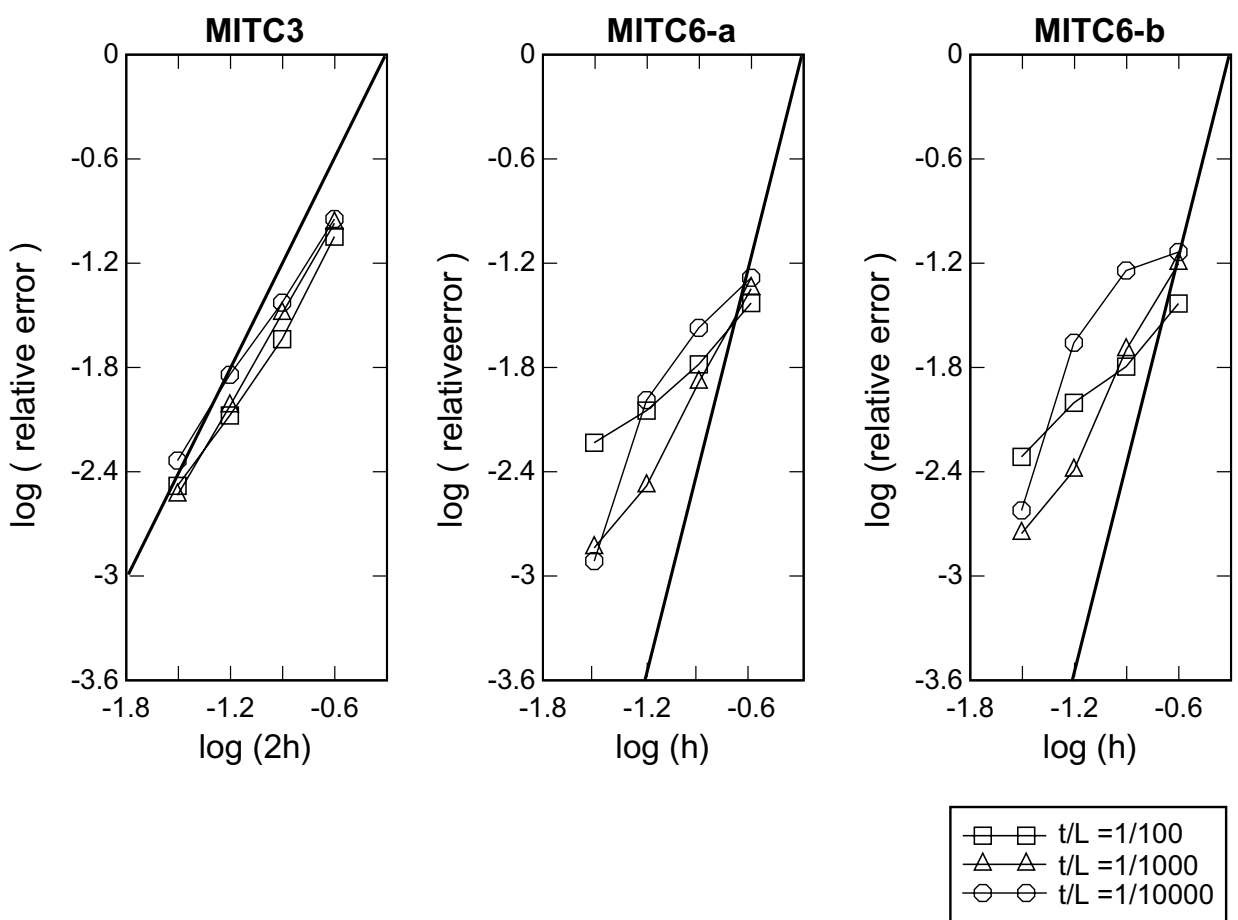

Fig. 15. Convergence curves for the clamped cylindrical shell problem. The bold line shows the optimal convergence rate, which is 2.0 for linear elements and 4.0 for quadratic elements.
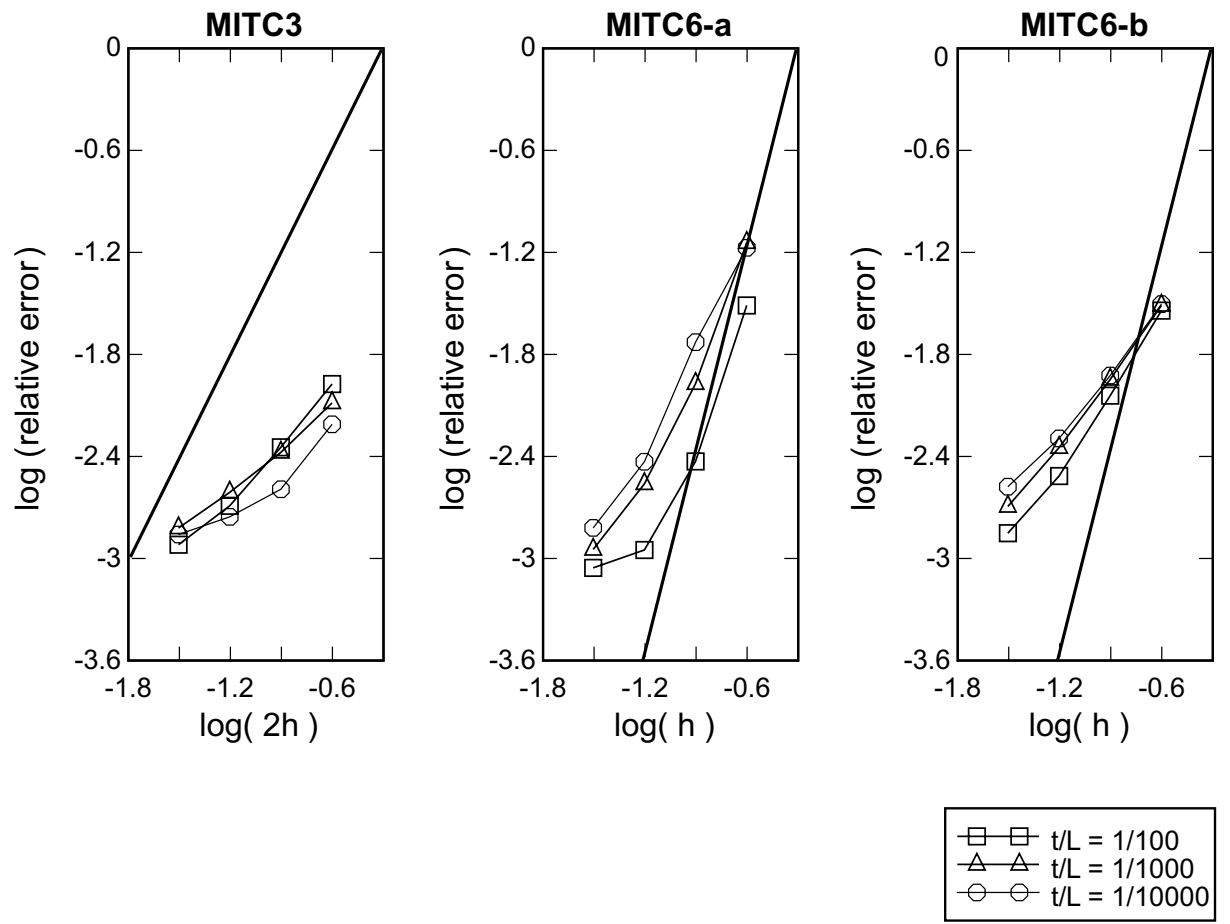

Fig. 16. Convergence curves for the free cylindrical shell problem. The bold line shows the optimal convergence rate, which is 2.0 for linear elements and 4.0 for quadratic elements. 

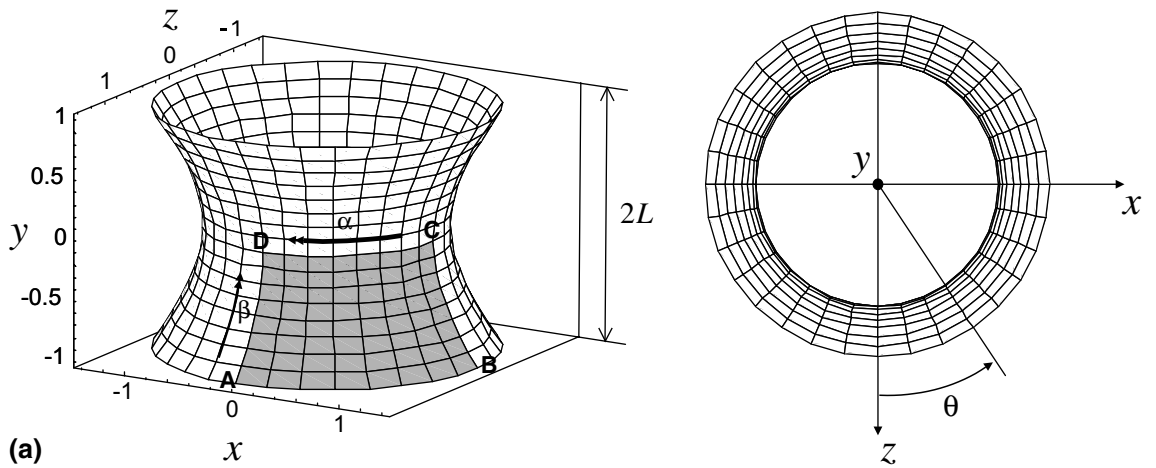

(b)

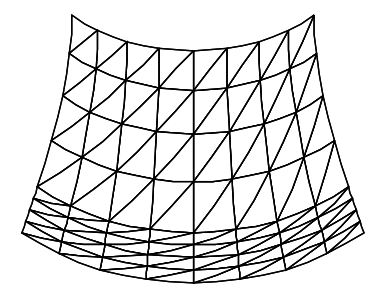

Fig. 17. (a) Hyperboloid shell problem $\left(E=2.0 \times 10^{11}, v=1 / 3\right.$ and $\left.p_{0}=1.0\right)$ and (b) graded mesh $(8 \times 8, t / L=1 / 1000$, clamped case).
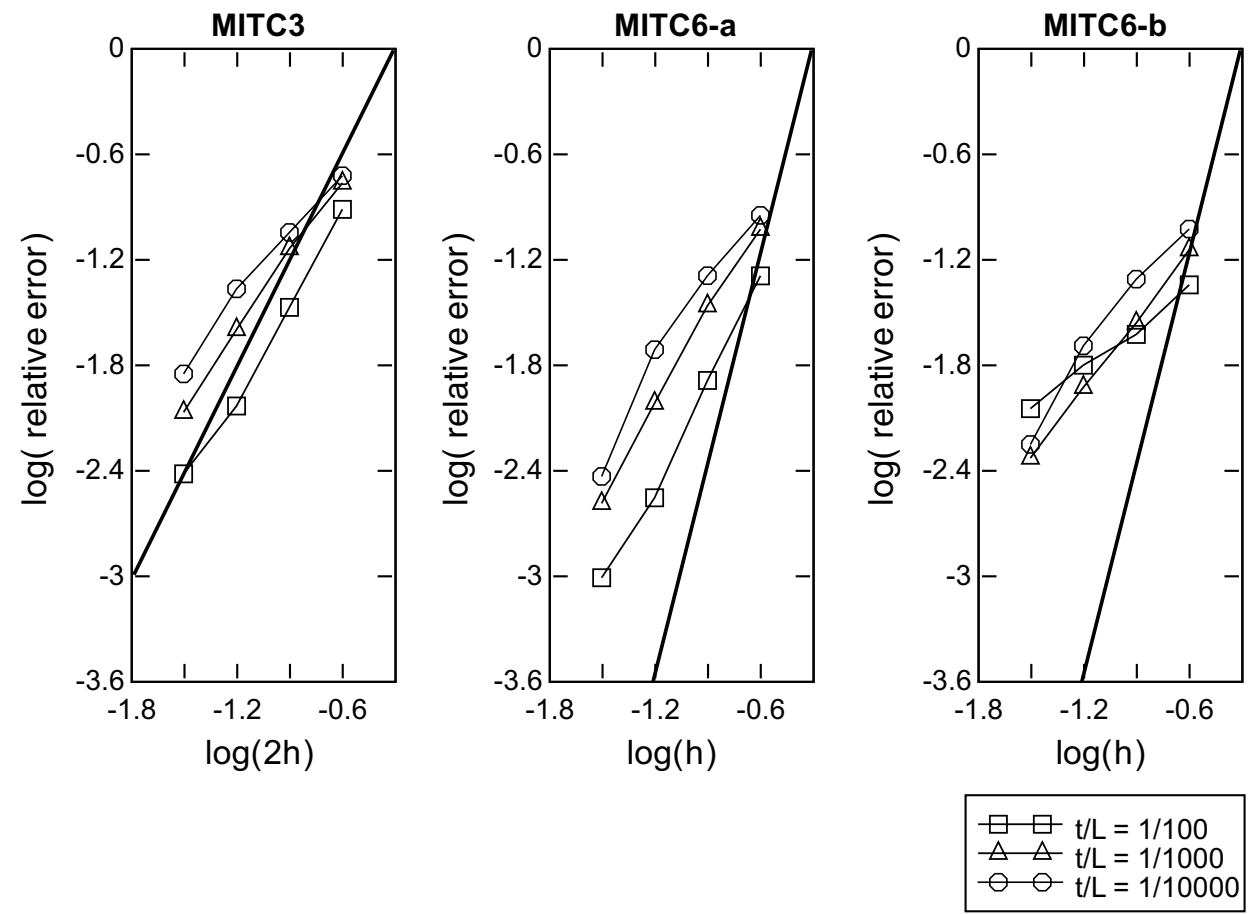

Fig. 18. Convergence curves for the clamped hyperboloid shell problem. The bold line shows the optimal convergence rate, which is 2.0 for linear elements and 4.0 for quadratic elements.

For both cases, we use the reference solution calculated using a mesh of $96 \times 96$ MITC9 shell elements. For the clamped case, half the mesh is used in the boundary layer of width $6 \sqrt{t}$, see Fig. 17(b). For the free case, the very thin boundary layer was not specially meshed.

Fig. 18 shows the convergence curves of the MITC triangular shell elements in the clamped case. 

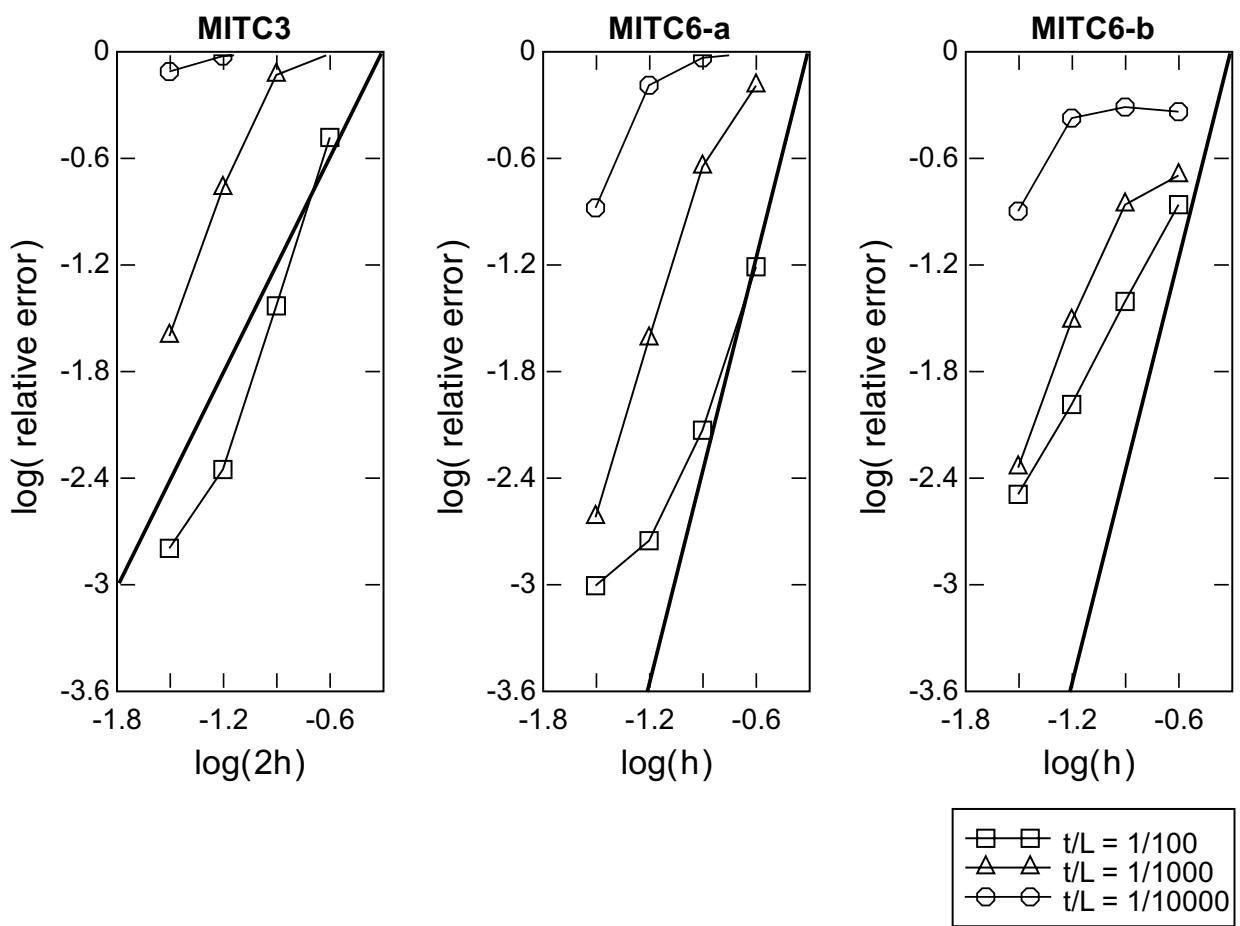

Fig. 19. Convergence curves for the free hyperboloid shell problem. The bold line shows the optimal convergence rate, which is 2.0 for linear elements and 4.0 for quadratic elements.

The MITC3 and MITC6-a elements show quite good convergence for this membrane dominated shell problem.

The convergence curves when the edges of the structure are free are shown in Fig. 19. This is a difficult problem to solve when the thickness is small [21], but the problem is an excellent test case because of the negative Gaussian curvature of the shell surface. The elements show all some locking but in fact good accuracy characteristics for the practical range of $t / L$ up to about $1 /$ 1000.

\section{Conclusions}

In this paper, we proposed a systematic procedure to construct spatially isotropic MITC triangular shell finite elements. The method is mechanically clear as well as simple and effective. We then constructed 3-node and 6node MITC shell finite elements. For the selected elements (the MITC3, MITC6-a and MITC6-b elements), we performed well-chosen numerical tests and showed convergence curves. While the elements have been developed and tested using the continuum-mechanics based approach with the Reissner-Mindlin kinematics (with the underlying basic shell model identified by
Chapelle and Bathe [2,22]), the same interpolation approach is of course also applicable to $3 \mathrm{D}$-shell elements [23].

The three elements considered are good candidates for the analysis of general shell structures in engineering practice in which the range of $t / L$ is usually from $1 / 10$ to about $1 / 1000$. The elements show good behavior in the chosen test problems for that range of thickness values. However, it is still necessary to study these elements further and to obtain uniformly optimal triangular shell finite elements that behave equally well in all types of shell problems.

\section{References}

[1] Bathe KJ. Finite element procedures. New York: Prentice Hall; 1996.

[2] Chapelle D, Bathe KJ. The finite element analysis of shells-fundamentals. Berlin: Springer-Verlag; 2003.

[3] Chapelle D, Bathe KJ. Fundamental considerations for the finite element analysis of shell structures. Comput Struct 1998;66:19-36, 711-712.

[4] Lee PS, Bathe KJ. On the asymptotic behavior of shell structures and the evaluation in finite element solutions. Comput Struct 2002;80:235-55. 
[5] Bathe KJ, Chapelle D, Lee PS. A shell problem 'highly sensitive' to thickness changes. Int J Numer Methods Eng 2003;57:1039-52.

[6] Dvorkin EN, Bathe KJ. A continuum mechanics based four-node shell element for general nonlinear analysis. Eng Comput 1984;1:77-88.

[7] Bathe KJ, Dvorkin EN. A formulation of general shell elements - the use of mixed interpolation of tensorial components. Int J Numer Methods Eng 1986;22:697722.

[8] Bucalem ML, Bathe KJ. Higher-order MITC general shell elements. Int J Numer Methods Eng 1993;36:3729-54.

[9] Bathe KJ, Brezzi F, Cho SW. The MITC7 and MITC9 plate bending elements. Comput Struct 1989;32:797-814.

[10] Bucalem ML, Nóbrega SHS. A mixed formulation for general triangular isoparametric shell elements based on the degenerated solid approach. Comput Struct 2000;78: $35-44$.

[11] Bathe KJ, Iosilevich A, Chapelle D. An inf-sup test for shell finite elements. Comput Struct 2000;75:439-56.

[12] Bathe KJ, Iosilevich A, Chapelle D. An evaluation of the MITC shell elements. Comput Struct 2000;75:1-30.

[13] Bathe KJ. The inf-sup condition and its evaluation for mixed finite element methods. Comput Struct 2001;79:24352, 971.

[14] Hiller JF, Bathe KJ. Measuring convergence of mixed finite element discretizations: an application to shell structures. Comput Struct 2003;81:639-54.
[15] Bletzinger KU, Bischoff M, Ramm E. A unified approach for shear-locking-free triangular and rectangular shell finite elements. Comput Struct 2000;75:321-34.

[16] Argyris JH, Papadrakakis M, Apostolopoulou C, Koutsourelakis S. The TRIC shell element: theoretical and numerical investigation. Comput Methods Appl Mech Eng 2000;182:217-45.

[17] Bernadou M, Eiroa PM, Trouvé P. On the convergence of a discrete Kirchhoff triangle method valid for shells of arbitrary shape. Comput Methods Appl Mech Eng 1994; 118:373-91.

[18] Sze KY, Zhu D. A quadratic assumed natural strain curved triangular shell element. Comput Methods Appl Mech Eng 1999;174:57-71.

[19] Kim JH, Kim YH. Three-node macro triangular shell element based on the assumed natural strains. Comput Mech 2002;29:441-58.

[20] Ahmad S, Irons BM, Zienkiewicz OC. Analysis of thick and thin shell structures by curved finite elements. Int J Numer Methods Eng 1970;2:419-51.

[21] Bathe KJ, Lee PS, Hiller JF. Towards improving the MITC9 shell element. Comput Struct 2003;81:477-89.

[22] Chapelle D, Bathe KJ. The mathematical shell model underlying general shell elements. Int $\mathbf{J}$ Numer Methods Eng 2000;48:289-313.

[23] Chapelle D, Ferent A, Bathe KJ. 3D-shell elements and their underlying mathematical model. Math Models Methods Appl Sci 2004;14:105-42. 